\title{
Connection Knowledge System for the Assembly Sequence Planning
}

\author{
Yin Wensheng \\ School of Mechanical Science and Engineering, \\ Huazhong University of Science and Technology, Wuhan 430074, China \\ wsyin@mail.hust.edu.cn
}

\begin{abstract}
Key words: assembly design; assembly sequence planning; connection; knowledge system Abstract. The design process of product is a process that uses engineering connection knowledge to assemble each functional unit, and the essence of which is the application of assembly connection knowledge. In the development of assembly design system which based on the knowledge of engineering connection, the assembly sequence planning is considered, and a new method of design and application of knowledge system is put forward. Firstly, the basic engineering connection relationship is analyzed, and the Problem Oriented Analysis and Decision Expert System (POADES) method is used to construct the knowledge mainframe tree, and then the basic connection classes (namely the connection nodes) are determined to realize the mapping relationship between the assembly model and the mainframe tree of connection knowledge. Finally, in order to provide convenience for the assembly sequence planning, the map data and the assembly sequence planning data are attached to the nodes.
\end{abstract}

\section{Introduction}

The basic operation of the assembly design system is to build the assembly model, that is, to use the engineering connection knowledge to assembly the different components of the product to make a product. The assembly design system building with this principle is called the assembly design system based on the knowledge of engineering connection. Assembly sequence planning is a very important task in the assembly design system. CAP (Connected Assembly Planning), which is designed and developed by the author, is a kind of assembly design system based on the knowledge of engineering connection. It can provide support for all kinds of assembly analysis, including assembly sequence planning. Since the system is based on the knowledge of engineering connection, it is very important to construct a connection knowledge system in this system.

\section{Related Work}

The construction of assembly design system and its connection knowledge system mainly involves two aspects. On the one hand, you must choose a suitable assembly model. On the other hand, you must choose an appropriate knowledge system.

Assembly Model. Bourjault first proposed a correlation model with graph expression and defined the physical contact relationship between the parts as connection, namely assembly relation [1]. The model is also called the assembly connection graph, which is based on the geometry of the parts. In order to improve this model, De Fazio, Whitney and Baldwin reduced the number of questions which needed to answer by the user [2]. Homem de Mello and Sanderson proposed that the assembly problem could be transformed into disassembly problem and then be solved with the method of cut set [3].

In order to avoid the combination explosion problem, Lee Sukhan proposed the assembly planning method based on the extraction of the subassembly [4]. Su Qiang divided the assembly design into the two layers of assembly model and hierarchical ASP [5]. Wang Yong divided complex products into a simple sub assembly planning structure in accordance with the requirements of the assembly [6]. Huang and Lee generated the priority relationship between parts according to the related process knowledge and algorithm [7].

The assembly model can also be described by matrix representation. For example, Dini and Santochi express product by the interference matrix, contact matrix and connection matrix [8]. Huang 
merges the 6 interference matrix into a disassembly matrix [9]. In addition, it can be described in the form of features.

Connecting Knowledge Model. At present, the assembly model used in the commercial assembly design system is a kind of model attaching mate relationships etc. to the product structure. This model can effectively record the composition and geometry information of the product, but it is difficult to solve many assembly problems, such as the combination explosion problem of assembly sequence planning. In order to improve the ability of the assembly design system, we must add the connection knowledge to the model.

For example, Yin et al. proposed the hierarchical model CBS and the connection relationship model CBRM, that is, CBS is generated by CBRM, and then the assembly sequence planning is performed by using CBS [10]. Zhang Gang et al. use knowledge to represent the assembly feature and solve the problem [11]. Dong Tianyang established an expression of the parts which based on the knowledge of the connection parts and used the knowledge of assembly sequence to solve the assembly sequence planning [12]. Li Rong, by analyzing the characteristics of the parts of the assembly, put forward the concept of the connection structure and built a Petri net [13].

Problem Oriented Analysis and Decision Expert System Method.Generally, the knowledge system is constructed by the method of expert system. The advantage is that it can make full use of the knowledge of experts. The disadvantage is that the workload of constructing the expert system is relatively large, the application scope is narrow, and it is difficult to be integrated with the assembly model. POADES is a system which is based on the core of the problem, according with the human thinking habits and application of knowledge, using the knowledge representation that support problem analysis and decision-making and take the problem object as the center. The knowledge unit includes 5 kinds of objects: problem object, meta-object, phenomenon, principle knowledge object and process knowledge object, that is, MP4 object. POADES uses the problem object as the main body to construct the knowledge mainframe tree, namely knowledge system, and its operation is simple, the knowledge capacity is big, the constructing and the maintenance is convenient [14-15], and can also be connected with the assembly model through the additional node data. Therefore, the author uses POADES to develop the connection knowledge system in CAP.

\section{Basic Engineering Connection Analysis}

Products are assembled through the engineering connection. This kind of engineering connection is the embodiment of the engineering assembly method which the enterprise can adopt in the process of product assembly. In the construction of a connection knowledge system, the relationships of engineering connection are required to be preserved in the form of knowledge base, so as to make use of the product design.

In the design of the connection knowledge system, the rationality of organization and the convenience of the use about engineering connection are considered. For example, the organization of engineering connection knowledge should be consistent with national standards. The engineering connection relation should be named with the method name of actual assembly operation. Mainly consider the following connection relationship:

1) Connection

According to the definition of the assembly model that based on the connection, the operation of connecting a number of components is called as connection. Here the connection relationship is the focal point. A connection can have a connector or not, and it can also be a combination of multiple connectors. 
2) Static connection and dynamic connection

Static and dynamic connection are important classification of the connection, and they have important function in the function, engineering design and manufacture of product and so on, and be seen as the first classification condition of the connection. The research is carried out in accordance with the static and dynamic connection.

3) The detachable connecting and no-detachable connection

The detachable connecting and no-detachable connection are important classification of connection in mechanical engineering.

4) Three basic fit connection relations

The three kinds of connection, that is the clearance fit, the transitional fit, and the interference fit, are the connection relations described in the fit and have particularity. The CAP system provides that the clearance fit belongs to the dynamic connection, and the interference fit belongs to the static connection, while the transitional fit is assumed to be able to define the movement between the two components, so it belongs to the static connection.

5) Common connections in mechanical engineering

In the manual of mechanical engineering, the standard part mainly includes connecting parts and non connecting parts. The former is mainly used to realize static connection, and the latter includes the bearing, gear and other dynamic connection components. In the connection knowledge system, the main concern is connection knowledge not the working principle and function of the dynamic connection components, and the key is to describe the relationship between the components and their relationship.

It is worth noting that, in the process of constructing the knowledge system of the engineering connection, because of the need to use a tree structure to express the network knowledge, it will appear many unknown and even contradictory. Especially, the typical situation is that in some cases, it is difficult to strictly distinguish static connection and dynamic connection. For example, the transition fit is generally classified as a static connection, but it may also be a relative motion. The pre tightening force also determines whether to produce the relative motion of the connected components when a bolt is connected, and so on.

\section{Engineering Connection Knowledge Mainframe Tree}

In the CAP system, the engineering connection knowledge mainframe tree is adopted as the knowledge representation. The core is to save the common engineering connection knowledge with the MP4 objects and its relations to the connection knowledge base.

Overall Architecture and Principles. The knowledge mainframe tree of engineering connection is a description and application of connection knowledge, which must be able to include the common engineering connection relationships. It also can be easily connected with the assembly model of CAP system.

Fig. 1 is a part of the connection knowledge mainframe tree of the CAP system. The main basis is as follows:

I The whole knowledge mainframe tree is related to connection knowledge, so the root node is named as "connection", while other nodes, in order to facilitate the description, are omitted "connection" word.

I In the upper level of knowledge mainframe tree, organizing the relationship with classification, so the relationship between them is Or relationship. While in the lower level of knowledge mainframe tree, organizing the relationship with structure, so the relationship between them is And relationship.

I The second layer mainly includes three kinds of connection relations, dynamic connection, static connection and typical connection. The typical connection is a common method in the enterprise, and it is the application of the dynamic connection and the static connection. 
I Defining basic connection classes. The basic connection class is the class which can reflect the characteristics of engineering connection. The basic connection class is corresponding to the assembly model of the CAP system.

I Connector model. It is the concrete component in the connection and must meet the national standards.

I The sub nodes of the basic connection classes should reflect the component objects and features that are involved in the connection.

I The typical connection also has a certain generality and can adapt to a wide range of enterprises.

I The extension section also includes a custom connection, which stores the connection of the designer's own definition.

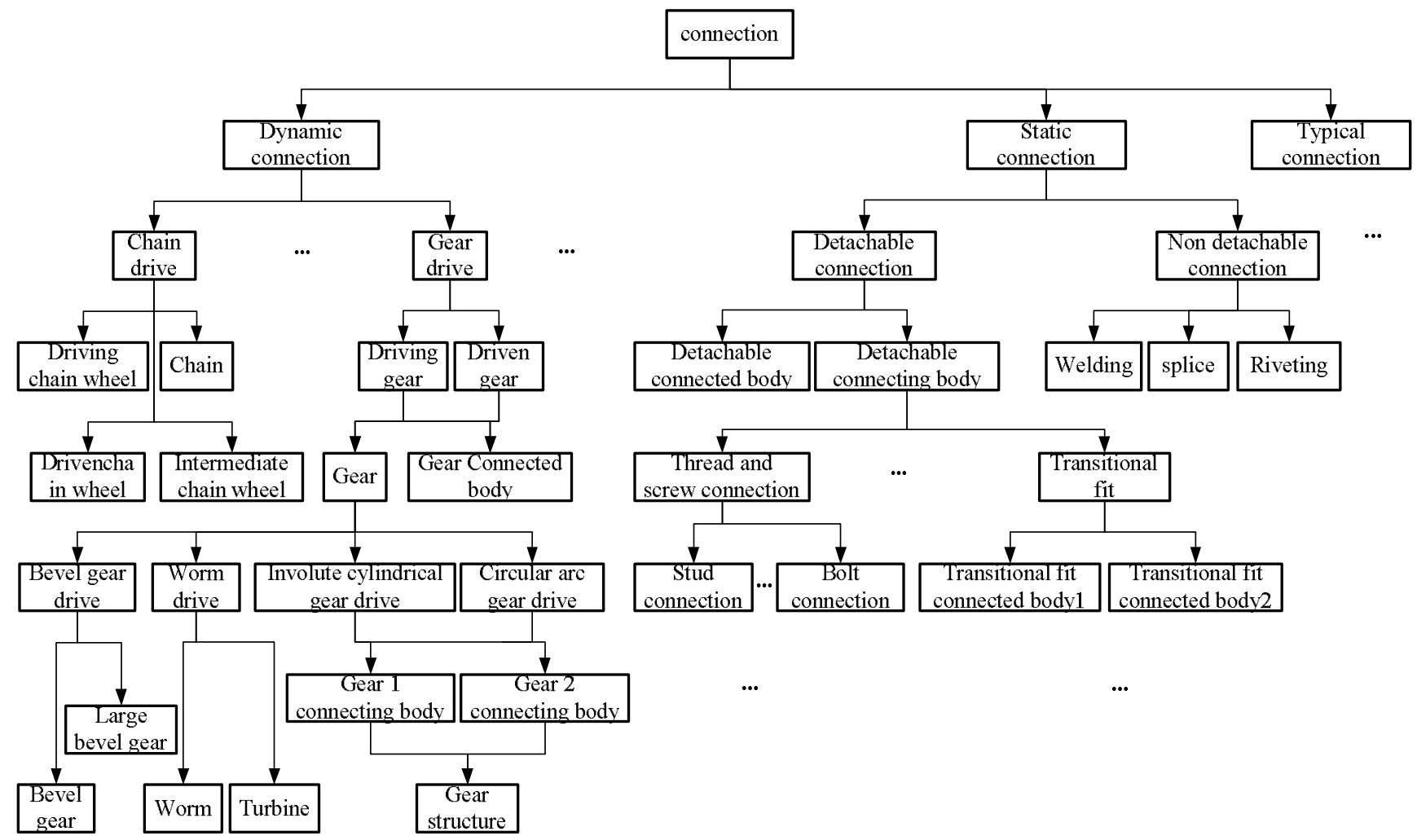

Fig. 1 the Knowledge Mainframe Tree of Engineering Connection

Basic Connection Class. The basic connection class reflects the characteristics of engineering connection and corresponds with connection nodes of the assembly model. At the same time, the basic connection class is the basis for the exchange among all kinds of person, so it should be defined as much as simple and clear.

The first layer and the second layer are obviously not as the basic connection class and only the third and below can be defined as the basic connection class.

The choice of basic connection class has the following principles:

I Have clear engineering meanings

I Be easy to distinguish

I Reduce the additional data

After the choice of basic connection class, the component objects should need to be determined to meet the requirement of the basic connection class. These objects are all the components involved in the design of the connection, which can be divided into two categories: connected and connector objects. Different basic connection classes have different definitions of connected and connector 
components. In general, the connected components are the functional component of product, not only their shape and structure change, but also the number changes.

The basic connection class is set up by analyzing the static connection, dynamic connection, and typical connection. For static connection, the components can be divided into two categories: one is the connection part which is also called the connector; the other is the functional component, which is called the connected object. For the dynamic connection, it is necessary to give the connection mode of each connection. For example, the components of the bearing connection are divided into three parts: the bearing inner ring, the bearing outer ring, and the bearing. There are two kinds of bearing expression, one is the decomposition mode, as shown in Fig. 2 (a) and the other one is the whole mode, as shown in Fig. 2 (b).

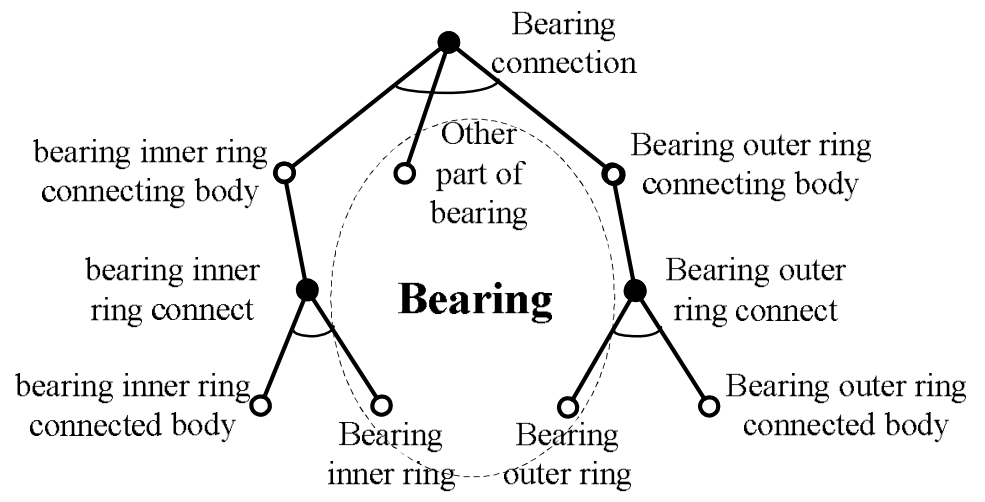

(a)

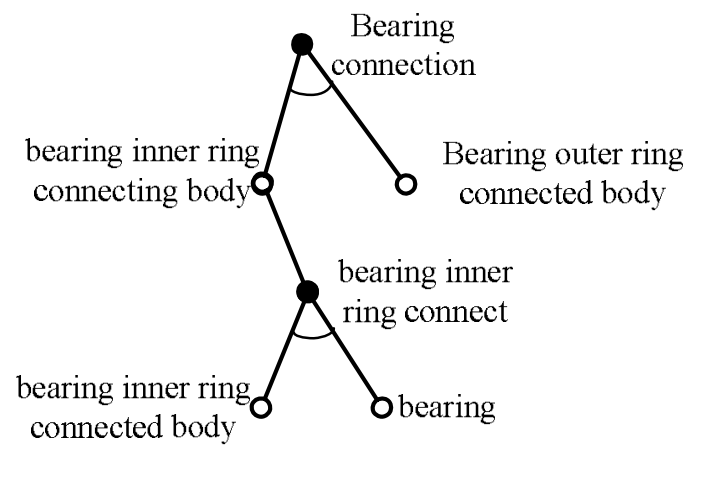

(b)

Fig. 2 Typical Connection Modes of Bearing

For gear drive, the participant components include "driving gear and driven gear" two parts, and each part can decomposition for "connected body" and "gear" in two parts. As shown in Fig. 3.

In addition, there are some special connections, such as the screw connection, which can not be used as the basic connection class. Only the concrete screw connection can be defined as a basic connection class. For example, you can define the common screw connection in mechanical engineering design manual as the basic connection mode.

Concrete Model Connection. When the user selects the basic connection class, the class of connection is determined fundamentally, but it needs to select the final connected parts and the connector, in particular, to select the connector meeting the requirements of national standards, industry standards and enterprise standards. The selection of the connector includes two parts, the selection of connector type and the connector dimension.

Meta-Object. In the connection knowledge, the meta-object is the dependent object of the connection relationship. In knowledge inference, the meta-object is often used to search for the relevant knowledge, so some abstract concepts can also be defined as the meta-object. 
The meta-object has the following categories.

I Various connectors, standard parts

Each kind of connection parts and standard parts are meta-object.

I Basic connection class

The basic connection class expresses the characteristics of the connection, which is important for the knowledge search.

I Abstract conception

Some abstract concepts can be used as meta-object. For example, some classification nodes connected to the knowledge mainframe tree can be set as the meta-object.

It is worth noting that, in order to retrieve conveniently, there should not be lot of connection knowledge having relation with the meta-object. Generally there are only 2 layers of nodes having relationship with the meta-object.

\section{Other MP4 Objects.}

Phenomenon object. Because the characteristics of the connection class in connection knowledge mainframe tree is obvious, so that the phenomenon object of the CAP system is not defined. The advantage of this is that the system is simple, and the disadvantage is that the retrieval and automatically inference can't be carried out by the phenomena.

Principle knowledge object. Principle knowledge object is organized with tree. The main goal is to convert a variety of mechanical design engineering manuals and national standards into the electronic one, that is, to store the national standards and other authority.

In CAP system, not only can refer to the knowledge in mainframe tree, but also can refer to a variety of mechanical design engineering manuals, national standards and other related mechanical design manual, e-books, etc.

Process knowledge object. The process knowledge object also uses the tree to organize the knowledge to describe the connection operation process of whole or part of product.

\section{Additional Data Connection}

The design and analysis of the product can be carried out effectively by attaching some data to the connection node.

Degree of Freedom. If the connection is not to be removed, the relative movement between the various components of the connection is not generated, and the freedom of each of them is 0 . However, if the connection can be removed, either the dynamic connection or the static connection, some of the components of the connection may generate relative movement to the other components in a certain force.

According to the function of freedom, it can be divided into two types, mechanism degree of freedom and the assembly degree of freedom. For assembly sequence planning, the assembly degree of freedom is recorded mainly.

Assembly Sequence Data. Assembly sequence data is generally attached to the basic connection class node in the connection knowledge mainframe tree. It mainly includes the connection class, the connection sub-components table and the assembly sequence table. And the connection class and the connection sub-components table is used to determine the object which participates in the connection operation, while the assembly sequence table records APG (Assembly Precedence Graph) data. Because of the need to establish the corresponding relationship between the connection knowledge system and the assembly sequence table, the connection class and the connection sub-components of the two systems must be consistent.

The definition of the assembly sequence table data is relatively simple, and the user only records the successively relationship between the two nodes. The system will automatically create an APG map according to the data. 


\section{Application and Maintenance of Connection Knowledge}

In the CAP system, the assembly design process is the process of constructing the connection node in the assembly model according to the connection knowledge system, namely the application process of connection knowledge. The basic method of the application process is:

Step 1: According to the engineering requirements, a suitable connection node is found in the connection knowledge mainframe tree and selected as the current node. The system copies the data into the current assembly model of the CAP system.

Step 2: According to the node data to determine the current connection object. When the assembly sequence planning is carried out, the system will prompt which object needed to be determined. The corresponding relationship between CAP and CAAD system is determined by selecting the corresponding components in the two systems.

Step 3: Repeat step 2 until all of the data is complete. At this point, the assembly sequence data is available.

In the matching process, some components can be added according to the actual situation, for example, the number of connected parts can be increased.

For better assembly design scheme, it can be written into the connection knowledge system. The process is called the maintenance of the connected knowledge system, and the steps are as follows:

Step 1: If a new APG data is relatively good, then find the corresponding node in the knowledge mainframe tree, and then test whether there is a corresponding component in the sub-component table, if there is one, turn to step 3.

Step 2: Add new sub component nodes and their sub - component tables in the connection knowledge system.

Step 3: Write the new assembly sequence data to the connection knowledge node. There are two ways, that is, cover and create.

\section{Conclusions}

Product design process is a process that uses engineering connection knowledge to assemble each functional unit, the essence of which is the application of assembly connection knowledge. A powerful assembly design system should not only provide the support of half structure or unstructured document to the connection design of the product, but also to be able to integrate these knowledge into the system, to guide or control the application of knowledge. It not only can simplify the assembly design process, but also to meet the actual product design better.

\section{References}

[1] Bourjault A. Contribution to a methodological approach of automated assembly: automatic generation of assembly sequence [D]. Besancon, France : University of Franchecomte, 1984 (in French)

[2] Delchambre A. Computer-aided assembly planning [M]. London, UK: Chapman \& Hall; 1992

[3] Homem de Mello L S, Sanderson A C. A correct and algorithm for the generation of mechanical assembly sequences [C]. IEEE Trans Robot Autom 1991, 7(2) : 228-40

[4] Sukhan Lee. Subassembly identification and evaluation for assembly planning [C]. IEEE Transaction on System, Man, and Cybernetics, 1994, 24(3) : 493-503

[5] Qiang Su. A hierarchical approach on assembly sequence planning and optimal sequences analyzing [J]. Robotics and Computer-Integrated Manufacturing 2009, 25:224-234

[6] Wang Yong, Liu Jihong. Assembly Unit Partitioning for Collaborative Assembly Planning [J]. Journal of Mechanical Engineering, 2009, 45(10) : 172-179 
[7] Huang Y F, Lee C S. A framework of knowledge-based assembly planning [C]. IEEE int. Conf. Roboties \& Automation. Sacramento, CA, U.S.A, 1991.4 : 599-604

[8] Dini, G, Santochi M. Automated sequencing and subassembly detection in assembly planning [C]. Annals of the CIRP, 1992, $41: 1-4$

[9] Huang Y M, Huang C T. Disassembly matrix for disassembly processes of products [J]. International Journal of Production Research, 2002, 40 (2) : 255-273

[10] Yin ZhouPing, Ding Han , Li HanXiong, et al. A connector-based hierarchical approach to assembly sequence planning for mechanical assemblies [J]. Computer Aided Design, 2003 , $35(1): 37-56$

[11]Zhang Gang, Yin Guofu, Deng Kewen, Cheng Erjing. Study on Assembly Sequence Planning Based on Knowledge [J]. Mechanical Science and Technology, 2006, 25(3) : 273-276

[12]Dong Tianyang, Tong Ruofeng, Zhang Ling, Dong Jinxiang. Assembly Sequence Planning Using Directed-Connector Knowledge [J]. Journal of Computer-aided Design \& Computer Graphics, 2004, 16(1) : 128-133

[13] Li Rong, Fu Yi-li, Feng Hai-bo. Assembly sequence planning based on connecter-structure knowledge [J]. Computer Integrated Manufacturing Systems, 2008, 14(6) : 1130-1135

[14] Wensheng Yin, Pinghui Tu, Xiuguo Chen, Hengxi Zhang. Problem Oriented Analysis and Decision Expert System with Large Capacity Knowledge-Base [C]. 2008 International Conference on Intelligent System and Knowledge Engineering (ISKE 2008), Xiamen, China, 2008.11:32 37

[15]YIN Wen-Sheng. Problem Oriented Analysis and Decision Expert System [J]. Application Research of Computers(in Chinese), 2008,25(12):3645-3649,3653 